\title{
Antiproliferative activity and synthesis of 8-prenylnaringenin derivatives by demethylation of 7-O- and $4^{\prime}-O$-substituted isoxanthohumols
}

\author{
Mirosław Anioł • Anna Świderska • \\ Monika Stompor • Anna Katarzyna Żołnierczyk
}

Received: 10 August 2011/ Accepted: 17 December 2011

(C) The Author(s) 2012. This article is published with open access at Springerlink.com

\begin{abstract}
Several analogues of 7-O- and 4'-O-substituted isoxanthohumol and 8-prenylnaringenin, the strongest known phytoestrogen and potential anticancerogenic agent, were synthesized. Acyl, alkyl, and allyl derivatives of isoxanthohumol underwent the demethylation process using $\mathrm{MgI}_{2} \times$ $2 \mathrm{Et}_{2} \mathrm{O}$ in anhydrous THF with the yields of $61-89 \%$. Some of the compounds approached the international criteria of antiproliferative activity $(4 \mu \mathrm{g} / \mathrm{ml})$ for synthetic agents against the human cancer cell lines.
\end{abstract}

Keywords Hop flavonoids - Phytoestrogens ·

Antitumor agents - Antiproliferative activity .

MCF-7 - HT-29 - CCRF/CEM

\section{Introduction}

Hops (Humulus lupulus L.) are used in the brewing industry to add flavor and bitterness to beer. They consist of many prenylated chalcones and flavanones (Stevens and Page, 2004). Among them, xanthohumol (1) has received much attention in recent years as an anti-cancer (Colgate et al., 2007; Drenzek et al., 2011; Okano et al., 2011), antioxidant (Delmulle et al., 2006; Jacob et al., 2011), and anti-HIV (Cos et al., 2008) agent. It is readily accessible from carbon dioxide-extracted-hops (spent hop) where its content ranges up to $1 \%$ of dry matter. Spent hop is an important by-product of the process of hop extraction in the beer brewing industry, which is usually used as a fertilizer

M. Anioł $(\varangle) \cdot$ A. Świderska · M. Stompor · A. K. Żołnierczyk Department of Chemistry, Wrocław University

of Environmental and Life Sciences, Norwida 25,

50-375 Wrocław, Poland

e-mail: Miroslaw.Aniol@wnoz.up.wroc.pl or as an animal feed in the U.S. However, in order to increase the added value of spent hops, hop processing industries have been looking for an alternative utilization of spent hops (Faltermeier et al., 2006; Oosterveld et al., 2002). Other flavonoids, isoxanthohumol (2) and 8-prenylnaringenin (3) are also present in hops, but in ten to one hundred times lower concentrations than the content of $\mathbf{1}$ (Stevens et al., 2000). Compound (3) is the potential drug in menopausal hormone therapy and the strongest phytoestrogen known in the nature (Borrelli and Ernst, 2010; Böttner, 2008; Chadwick et al., 2006; Hyun et al., 2008; Overk et al. 2008). The compounds (1-3) have also antibreast cancer activity (Brunelli et al., 2007; Monteiro et al., 2007; Wesolowska et al., 2010a, b). Prenylflavonoid (3) can be synthesized in high yield from xanthohumol (1). It requires the cyclization of $\mathbf{1}$ to isoxanthohumol (2) in basic conditions and demethylation of $\mathbf{2 - 3}$ with $\mathrm{MgI}_{2} \times 2 \mathrm{Et}_{2} \mathrm{O}$ (Anioł et al., 2008).

Wilhelm and Wessjohann, (2006) studied demethylation of 2-3 with $\mathrm{AlBr}_{3}, \mathrm{BBr}_{3}$ or $\mathrm{MeAlCl}_{2}$ in collidine; $\mathrm{ZnBr}_{2}$, $\mathrm{CuI}, \mathrm{ZnBr}_{2} / \mathrm{CuI} \mathrm{Yb}_{2}\left(\mathrm{SO}_{4}\right)_{3} / \mathrm{KI}$ or $\mathrm{CuI}, \mathrm{Sm}(\mathrm{OTf})_{3} / \mathrm{KI}$, $\mathrm{CeCl}_{3} / \mathrm{LiI}$. Product (3) was not detected or obtained with low yield. Hydroxyl groups of $\mathbf{2}$ were also protected with chlorotriisopropylsilane, demethylated with $\mathrm{AlBr}_{3}$ and deprotected with $(n-\mathrm{Bu})_{4} \mathrm{NF}$ to obtain 8-prenylnaringenin (3) with $73 \%$ yield. The best result was obtained for $\mathrm{Sc}(\mathrm{OTf})_{3} / \mathrm{KI}(92 \%)$.

Magnesium iodide etherate was previously applied in the regioselective demethylation of 5-acetyl-4,6-dimethoxy-2-isopropenyl-2,3-dihydrobenzofuran (Yamaguchi et al., 1987) and substituted 2,6-dimethoxybenzaldehydes (Yamaguchi et al., 1999).

Only a few studies can be found in the literature that reported 8-prenylnaringenin and isoxanthohumol derivative synthesis. Methylation of 8-prenylnaringenin (3) with 
$\mathrm{Me}_{2} \mathrm{SO}_{4}$ resulted in the formation of di- $O$-methyl derivatives of 1 and 2 (Jain et al., 1978). The synthesis of $7,4^{\prime}$-di- $O$-acetyl-8-prenylnaringenin was carried out using $7,4^{\prime}$-di- $O$-acetylnaringenin as a substrate via its 4- $O$-prenyl ether, which undertook the Claisen-Cope rearrangement (Gester et al., 2001). The preparation of chiral 7,4'-dimethyl- or diacetyl- isoxanthohumols and 8-prenylnaringenins was achieved by reducing a carbonyl group to a hydroxyl group with a mixture of formic acid and a base in the presence of chiral catalyst. Separation of the non-transferred enantiomer $(2 \mathrm{~S})$ or $(2 \mathrm{R})$ of the reduced 8-prenylnaringenin diacetyl derivative and splitting the acyl residues in enantiomers by enzyme catalyst solvolysis gave (2S)-8prenylnaringenin or (2R)-8-prenylnaringenin. The second enantiomers $(2 \mathrm{R})$ or $(2 \mathrm{~S})$ of 8-prenylnaringenin diacetyl derivative was recovered by oxygenation of a hydroxyl group (Metz and Schwab, 2007). Starting from 3, several carboxylic acid haptenes of this compound were also synthesized. Five linkers [ $-\left(\mathrm{CH}_{2}\right)_{n} \mathrm{COOH}, n=1,3,5,6$, and 9] were coupled to the $\mathrm{C} 7-\mathrm{OH}$ or $\mathrm{C}^{\prime}-\mathrm{OH}$ group of 8-prenylnaringenin to obtain five derivatives (Schaefer et al., 2005).

In this article, we report methods of synthesis of 7-Oand $4^{\prime}-O$-substituted alkyl, alkenyl and acyl isoxanthohumol derivatives and their demethylation using magnesium iodide etherate. This research is connected with utilization of the spent hop, obtained after extraction with supercritical carbon dioxide. This waste product of the hop industry is rich in xanthohumol, the starting compound in the synthesis of all the compounds described in this article.

\section{Materials and methods}

\section{Chemistry}

\section{General}

All the reactions were carried out under a dry nitrogen atmosphere. The organic solvents were dried and purified according to the standard procedures. The reagents were purchased from Fluka. Isoxanthohumol (2) was obtained from xanthohumol (1) by dissolving in $1 \% \mathrm{NaOH}$ and acidification of the reaction mixture as it was described previously (Anioł et al., 2008). Analytical thin-layer chromatography was carried out on DC-Alufolien Kieselgel $60 \mathrm{~F}_{254}$ silica gel $(0.2 \mathrm{~mm}$; Merck) with chloroform: methanol (96:4) as the developing solvent. Visualization was effected with a solution of $10 \mathrm{~g} \mathrm{Ce}\left(\mathrm{SO}_{4}\right)_{2}$ and $20 \mathrm{~g}$ phosphomolybdic acid in 11 of $10 \% \mathrm{H}_{2} \mathrm{SO}_{4}$, followed by heating. Preparative column chromatography was accomplished using silica gel (Kiesel 60, 230-400 mesh; Merck) columns. Proton NMR spectra were recorded on a Bruker AMX 300 instrument at $300 \mathrm{MHz}$ with acetone- $\mathrm{d}_{6}$ as the solvent and TMS as an internal standard. The infrared (IR) spectra in $\mathrm{KBr}$ were recorded on a Mattson IR 300 spectrometer.

\section{Synthesis of isoxanthohumol derivatives}

7,4'-Di-O-methylisoxanthohumol (4) and 7-O-methylisoxanthohumol (5) A mixture of isoxanthohumol (100 $\mathrm{mg}, 0.282 \mathrm{mmol})$, anhydrous $\mathrm{K}_{2} \mathrm{CO}_{3}(232 \mathrm{mg}, 1.68 \mathrm{mmol})$, and methyl iodide $(0.5 \mathrm{ml})$ in $5 \mathrm{ml}$ of anhydrous acetone was stirred for $12 \mathrm{~h}$ at room temperature. Acetone was evaporated and the resultant reaction mixture was treated with $10 \mathrm{ml}$ of a saturated $\mathrm{NaCl}$ solution and extracted with $\mathrm{Et}_{2} \mathrm{O}(3 \times$ $10 \mathrm{ml}$ ). The organic phase was dried over anhydrous $\mathrm{Na}_{2} \mathrm{SO}_{4}$, concentrated and was subjected to column chromatography $\left(\mathrm{CHCl}_{3}: \mathrm{MeOH}, 99: 1\right)$ to provide $74.9 \mathrm{mg}(69.4 \%)$ of light yellow solid $\left(\mathrm{mp}=37-39^{\circ} \mathrm{C}, R_{\mathrm{f}}=0.60, \mathrm{CHCl}_{3}: \mathrm{MeOH}\right.$, 98:2) of 7,4'-di- $O$-methylisoxanthohumol (4) and $9.1 \mathrm{mg}$ $(8.8 \%)$ of white solid $\left(\mathrm{mp}=181-184^{\circ} \mathrm{C}, R_{\mathrm{f}}=0.21, \mathrm{CHCl}_{3}\right.$ : $\mathrm{MeOH}, 98: 2)$ of 7-O-methylisoxanthohumol (5). ${ }^{1} \mathrm{H}$ NMR and IR spectroscopic data were in agreement with those reported in the literature (Metz and Schwab, 2007; Stevens et al., 2000).

7-O-n-pentylisoxanthohumol (6) and 7,4'-di-O-n-pentyl-8isoxanthohumol (7) The reaction was carried out exactly in the same way as it is described for compounds ( $\mathbf{4}$ and $\mathbf{5}$ ) but $1 \mathrm{ml}$ of $n$-pentyl iodide was used instead of methyl iodide. The product (33.5 mg, 27.6\%) 7-O-n-pentylisoxanthohumol (6) was obtained as a pale yellow solid $\left(\mathrm{mp}=140-142^{\circ} \mathrm{C}\right.$, $\left.R_{\mathrm{f}}=0.61, \mathrm{CHCl}_{3}: \mathrm{MeOH}, 97: 3\right)$. The ${ }^{1} \mathrm{H}$ NMR $(300 \mathrm{MHz}$, acetone- $d_{6}$ ) for compound $(\mathbf{6}): \delta(\mathrm{ppm}): 0.93(t, 3 \mathrm{H}$, $\left.J=7.1 \mathrm{~Hz}, \mathrm{C}-7-\mathrm{O}\left(\mathrm{CH}_{2}\right)_{4} \mathrm{CH}_{3}\right) ; 1.33-1.54(\mathrm{~m}, 4 \mathrm{H}, \mathrm{C}-7-$ $\left.\mathrm{O}\left(\mathrm{CH}_{2}\right)_{2} \mathrm{CH}_{2} \mathrm{CH}_{2} \mathrm{CH}_{3}\right) ; 1.61$ (d, $6 \mathrm{H}, J=1.3 \mathrm{~Hz}, \mathrm{CH}_{3}-4^{\prime \prime}$ and $\left.\mathrm{CH}_{3}-5^{\prime \prime}\right) ; 1.78-1.87\left(\mathrm{~m}, 2 \mathrm{H}, \mathrm{C} 7-\mathrm{OCH}_{2} \mathrm{CH}_{2}\left(\mathrm{CH}_{2}\right)_{2} \mathrm{CH}_{3}\right)$; $2.63(\mathrm{dd}, 1 \mathrm{H}, J=16.4 \mathrm{~Hz}, J=3.0 \mathrm{~Hz}, \mathrm{CH}-3) ; 2.93$ (dd, $1 \mathrm{H}, J=16.4 \mathrm{~Hz}, J=12.5 \mathrm{~Hz}, \mathrm{CH}-3) ; 3.26(\mathrm{~d}, 2 \mathrm{H}$, $\left.J=7.1 \mathrm{~Hz}, \mathrm{CH}_{2}-1^{\prime \prime}\right) ; 3.84$ (s, 3H, C-5-OCH $) ; 4.13(t, 2 \mathrm{H}$, $\left.J=6.3 \mathrm{~Hz}, \mathrm{C}-7-\mathrm{OCH}_{2}\left(\mathrm{CH}_{2}\right)_{3} \mathrm{CH}_{3}\right) ; 5.16\left(t_{\text {sept }}, 1 \mathrm{H}, J=\right.$ $\left.7.1 \mathrm{~Hz}, J=1.3 \mathrm{~Hz}, \mathrm{CH}-2^{\prime \prime}\right) ; 5.36(\mathrm{dd}, 1 \mathrm{H}, J=12.5 \mathrm{~Hz}$, $J=3.0 \mathrm{~Hz}, \mathrm{CH}-2) ; 6.34$ (s, 1H, CH-6); 6.89(d, 2H, $J=8.6 \mathrm{~Hz}, \mathrm{CH}-3^{\prime}$ and $\left.\mathrm{CH}-5^{\prime}\right) ; 7.38(\mathrm{~d}, 2 \mathrm{H}, J=8.6 \mathrm{~Hz}$, CH-2' i CH-6'); 8.53 (s, 1H, C-4'-OH). IR (KBr) cm ${ }^{-1}: 2957$, 2931, 2856, 1665, 1599, 1570, 1520, 1458, 1262, 1103, 798. $\mathrm{C}_{26} \mathrm{H}_{32} \mathrm{O}_{5}$ (424.54): calcd. C 73.56, H 7.60; found C 73.67, H 6.75. The compound 7,4'-di- $O-n$-pentyl-8-isoxanthohumol (7) was also isolated (18.4 mg, $13.6 \%$ yield) as a light yellow solid (mp $\left.=70-75^{\circ} \mathrm{C}, R_{\mathrm{f}}=0.87, \mathrm{CHCl}_{3}: \mathrm{MeOH}, 97: 3\right) .{ }^{1} \mathrm{H}$ NMR $\left(300 \mathrm{MHz}\right.$, acetone- $\left.d_{6}\right) \delta(\mathrm{ppm}): 0.93(t, 6 \mathrm{H}$, $\mathrm{J}=7.1 \mathrm{~Hz}, \mathrm{C}-7-$ and $\left.\mathrm{C}-4^{\prime \prime}-\mathrm{O}\left(\mathrm{CH}_{2}\right)_{4} \mathrm{CH}_{3}\right) ; 1.34-1.54(\mathrm{~m}$, $8 \mathrm{H}, \mathrm{C}-7$ - and $\left.\mathrm{C}-4^{\prime}-\mathrm{O}\left(\mathrm{CH}_{2}\right)_{2} \mathrm{CH}_{2} \mathrm{CH}_{2} \mathrm{CH}_{3}\right) ; 1.62(\mathrm{~d}, 6 \mathrm{H}$, $J=1.3 \mathrm{~Hz}, \mathrm{CH}_{3}-4^{\prime \prime}$ and $\left.\mathrm{CH}_{3}-5^{\prime \prime}\right) ; 1.74-1.87$ (m, 4H, C-7and $\left.\mathrm{C}^{\prime}-\mathrm{OCH}_{2} \mathrm{CH}_{2}\left(\mathrm{CH}_{2}\right)_{2} \mathrm{CH}_{3}\right) ; 2.65(\mathrm{dd}, 1 \mathrm{H}, J=16.3 \mathrm{~Hz}$, 
$J=3.0 \mathrm{~Hz}, \quad \mathrm{CH}-3) ; \quad 2.95 \quad(\mathrm{dd}, \quad 1 \mathrm{H}, \quad J=16.3 \mathrm{~Hz}$, $J=12.5 \mathrm{~Hz}, \mathrm{CH}-3) ; 3.28\left(\mathrm{~d}, 2 \mathrm{H}, J=7.1 \mathrm{~Hz}, \mathrm{CH}_{2}-1^{\prime \prime}\right)$; $3.84\left(\mathrm{~s}, 3 \mathrm{H}, \mathrm{C}-5-\mathrm{OCH}_{3}\right) ; 4.02\left(t, 2 \mathrm{H}, J=6.5 \mathrm{~Hz}, \mathrm{C}-4^{\prime}-\right.$ $\left.\mathrm{OCH}_{2}\left(\mathrm{CH}_{2}\right)_{3} \mathrm{CH}_{3}\right) ; 4.13\left(t, 2 \mathrm{H}, J=6.3 \mathrm{~Hz}, \mathrm{C}-7-\mathrm{OCH}_{2}\right.$ $\left.\left(\mathrm{CH}_{2}\right)_{3} \mathrm{CH}_{3}\right) ; 5.17\left(t_{\text {sept }}, 1 \mathrm{H}, J=7.1 \mathrm{~Hz}, J=1.3 \mathrm{~Hz}, \mathrm{CH}-\right.$ $\left.2^{\prime \prime}\right) ; 5.43(\mathrm{dd}, 1 \mathrm{H}, J=12.5 \mathrm{~Hz}, J=3.0 \mathrm{~Hz}, \mathrm{CH}-2) ; 6.34(\mathrm{~s}$, $1 \mathrm{H}, \mathrm{CH}-6) ; 6.98\left(\mathrm{~d}, 2 \mathrm{H}, J=8.7 \mathrm{~Hz}, \mathrm{CH}-3^{\prime}\right.$ and $\left.\mathrm{CH}^{\prime} 5^{\prime}\right) ; 7.46$ (d, $2 \mathrm{H}, J=8.7 \mathrm{~Hz}, \mathrm{CH}-2^{\prime}$ and $\left.\mathrm{CH}-6^{\prime}\right)$. IR $(\mathrm{KBr}) \mathrm{cm}^{-1}$ : 3064 , 2952, 2936, 2870, 1675, 1601, 1577, 1517, 1465, 1346, 1253, 1113, 827. $\mathrm{C}_{31} \mathrm{H}_{42} \mathrm{O}_{5}$ (494.68): calcd. C 75.27, H 8.56; found C 75.51, H 8.44.

7,4'-Di-O-allylisoxanthohumol (8) The reaction was carried out similarly as it is described for compounds ( 4 and 5 ) but $1 \mathrm{ml}$ of allyl bromide and $6 \mathrm{ml}$ of anhydrous THF were used instead methyl iodide and acetone. The product was purified by column chromatography $\left(\mathrm{CHCl}_{3}: \mathrm{MeOH}, 99.3: 0.7\right)$ to give $100.2 \mathrm{mg}$ of $7,4^{\prime}$-di- $O$-allylisoxanthohumol (8) as a light yellow solid $\left(\mathrm{mp}=79-83^{\circ} \mathrm{C}, R_{\mathrm{f}}=0.85, \mathrm{CHCl}_{3}: \mathrm{MeOH}\right.$, 95:5) with $81.2 \%$ yield. ${ }^{1} \mathrm{H}$ NMR (300 MHz, acetone- $\left.d_{6}\right) \delta$ (ppm): $1.61\left(\mathrm{~d}, 6 \mathrm{H}, J=1.4 \mathrm{~Hz}, \mathrm{CH}_{3}-4^{\prime \prime}\right.$ and $\left.\mathrm{CH}_{3}-5^{\prime \prime}\right) ; 2.66$ (dd, $1 \mathrm{H}, J=16.3 \mathrm{~Hz}, \mathrm{~J}=3.1 \mathrm{~Hz}, \mathrm{CH}-3$ ); 2.95 (dd, $1 \mathrm{H}$, $J=16.3 \mathrm{~Hz}, J=12.5 \mathrm{~Hz}, \mathrm{CH}-3) ; 3.28(\mathrm{~d}, 2 \mathrm{H}, J=7.2 \mathrm{~Hz}$, $\mathrm{CH}_{2}-1^{\prime \prime}$ ); 3.84 (s, 3H, C-5-OCH 3 ); 4.61 and 4.73 (two ddd, $4 \mathrm{H}, J=5.2 \mathrm{~Hz}, J=1.7 \mathrm{~Hz}, J=1.5 \mathrm{~Hz}, \mathrm{C}-7-$ and $\mathrm{C}-4^{\prime}-$ $\left.\mathrm{OCH}_{2} \mathrm{CH}=\mathrm{CH}_{2}\right) ; 5.18\left(t_{\text {sept }}, 1 \mathrm{H}, J=7.2 \mathrm{~Hz}, J=1.4 \mathrm{~Hz}\right.$, CH-2 ${ }^{\prime \prime}$ ); 5.25 and 5.29 (two dq, $2 \mathrm{H}, J=10.4 \mathrm{~Hz}, J=1.5 \mathrm{~Hz}$ and $J=10.4 \mathrm{~Hz}, J=1.5 \mathrm{~Hz}$, trans-C-7- and trans-C-4'$\left.\mathrm{OCH}_{2} \mathrm{CH}=\mathrm{CH}_{2}\right) ; 5.42(\mathrm{dd}, 1 \mathrm{H}, J=12.5 \mathrm{~Hz}, J=3.1 \mathrm{~Hz}$, $\mathrm{CH}-2$ ); 5.41 and 5.47 (two dq, $2 \mathrm{H}, J=8.8 \mathrm{~Hz}, 1.7 \mathrm{~Hz}$, $J=8.8 \mathrm{~Hz}, 1.7 \mathrm{~Hz}$, cis-C-7- and cis-C-4'- $\mathrm{OCH}_{2} \mathrm{CH}=\mathrm{CH}_{2}$ ); 6.09 and 6.11 (two ddt, $2 \mathrm{H}, J=10.4 \mathrm{~Hz}, J=8.8 \mathrm{~Hz}, 5.2 \mathrm{~Hz}$ i $J=10.4 \mathrm{~Hz}, J=8.8 \mathrm{~Hz}, 5.2 \mathrm{~Hz}, \mathrm{C}-7-$ i C-4'- $\mathrm{OCH}_{2}$ $\left.\mathrm{CH}=\mathrm{CH}_{2}\right) ; 6.36(\mathrm{~s}, 1 \mathrm{H}, \mathrm{CH}-6) ; 7.01\left(\mathrm{~d}, 2 \mathrm{H}, J=8.7 \mathrm{~Hz}, \mathrm{CH}-3^{\prime}\right.$ and $\left.\mathrm{CH}^{-} 5^{\prime}\right) ; 7.48\left(\mathrm{~d}, 2 \mathrm{H}, J=8.7 \mathrm{~Hz}, \mathrm{CH}-2^{\prime}\right.$ and $\left.\mathrm{CH}-6^{\prime}\right)$. IR $(\mathrm{KBr}) \mathrm{cm}^{-1}: 3080,2985,2962,2915,2852,1678,1604,1574$, $1515,1272,1116,1018,932,821 . \mathrm{C}_{27} \mathrm{H}_{30} \mathrm{O}_{5}$ (434.54): calcd. C 74.63, H 6.96; found C 74.70, H 7.02.

7,4'-Di-O-acetylisoxanthohumol (9) To a solution of $100 \mathrm{mg}(0.282 \mathrm{mmol})$ of isoxanthohumol and $0.37 \mathrm{ml}$ (2.8 mmol) of $\mathrm{Et}_{3} \mathrm{~N}$ in $7.4 \mathrm{ml}$ of anhydrous THF was added dropwise acetic anhydride $0.13 \mathrm{ml}, 1.4 \mathrm{mmol}$ ). After $12 \mathrm{~h}$ of stirring at room temperature, the reaction medium was shaken with $36 \mathrm{ml}$ of cooled water. The precipitated crystals were separated, washed twice with $3 \mathrm{ml}$ of water, and dried using vacuum. The crude product was purified by the crystallization from methanol to provide 7, $4^{\prime}$-di- $O$ acetylisoxanthohumol (9) as white crystals $(92.1 \mathrm{mg}$, $74.1 \%$ yield, $\mathrm{mp}=140-141^{\circ} \mathrm{C}, R_{\mathrm{f}}=0.68, \mathrm{CHCl}_{3}: \mathrm{MeOH}$, 99:1). ${ }^{1} \mathrm{H}$ NMR (300 MHz, acetone- $\left.d_{6}\right) \delta(\mathrm{ppm}): 1.58$ and $1.61\left(\mathrm{~d}, 6 \mathrm{H}, J=1.4 \mathrm{~Hz}, \mathrm{CH}_{3}-4^{\prime \prime}\right.$ and $\left.\mathrm{CH}_{3}-5^{\prime \prime}\right) ; 2.27(\mathrm{~s}, 3 \mathrm{H}$, C-4'- $\left.\mathrm{COOCH}_{3}\right) ; 2.31$ (s, 3H, C-7-COOCH 3$) ; 2.78(\mathrm{dd}, 1 \mathrm{H}$,
$J=16.3 \mathrm{~Hz}, J=3.1 \mathrm{~Hz}, \mathrm{CH}-3) ; 3.06(\mathrm{dd}, 1 \mathrm{H}, J=16.3$ $\mathrm{Hz}, J=12.9 \mathrm{~Hz}, \mathrm{CH}-3) ; 3.19\left(\mathrm{~d}, 2 \mathrm{H}, J=7.02 \mathrm{~Hz}, \mathrm{CH}_{2^{-}}\right.$ $\left.1^{\prime \prime}\right) ; 3.80\left(\mathrm{~s}, 3 \mathrm{H}, \mathrm{C}-5-\mathrm{O}-\mathrm{CH}_{3}\right) ; 5.09\left(t_{\text {sept }}, 1 \mathrm{H}, J=7.1 \mathrm{~Hz}\right.$, $\left.J=1.4 \mathrm{~Hz}, \quad \mathrm{CH}-2^{\prime \prime}\right) ; \quad 5.59 \quad(\mathrm{dd}, \quad 1 \mathrm{H}, \quad J=12.9 \mathrm{~Hz}$, $J=2.9 \mathrm{~Hz}, \mathrm{CH}-2) ; 6.49$ (s, 1H, CH-6); 7.21 (d, 2H, $J=8.6 \mathrm{~Hz}, \mathrm{CH}-3^{\prime}$ and $\left.\mathrm{CH}-5^{\prime}\right) ; 7.62(\mathrm{~d}, 2 \mathrm{H}, J=8.5 \mathrm{~Hz}$, $\mathrm{CH}-2^{\prime}$ and $\left.\mathrm{CH}-6^{\prime}\right)$. IR (KBr) $\mathrm{cm}^{-1}: 2964,2927,1759$, 1687, 1593, 1510, 1477, 1369, 1213, 1170, 1093, 837. $\mathrm{C}_{25} \mathrm{H}_{26} \mathrm{O}_{7}$ (438.48): calcd. C 68.48, H 5.98; found C 68.58, H 6.10 .

7,4'-Di-O-palmitoylisoxanthohumol (10) To a solution of $100 \mathrm{mg}(0.282 \mathrm{mmol})$ of isoxanthohumol and $0.28 \mathrm{ml}$ (2.1 mmol) of $\mathrm{Et}_{3} \mathrm{~N}$ in $5.7 \mathrm{ml}$ of anhydrous THF was added dropwise palmitoyl chloride $(155 \mathrm{mg}, 0.594 \mathrm{mmol})$. After $12 \mathrm{~h}$ of stirring at room temperature the reaction medium was shaken with $30 \mathrm{ml}$ of cold water $\left(\sim 0^{\circ} \mathrm{C}\right)$, extracted with diethyl ether $(3 \times 10 \mathrm{ml})$, dried over anhydrous $\mathrm{Na}_{2} \mathrm{SO}_{4}$, and concentrated. The resulting residue was purified by column chromatography (hexane: $\mathrm{Et}_{2} \mathrm{O}: \mathrm{MeOH}, 5: 5: 1$ ) to give 191.2 $\mathrm{mg}(81.6 \%$ yield) of 7,4'-di- $O$-palmitoylisoxanthohumol (10) as white crystals $\left(\mathrm{mp}=71-73^{\circ} \mathrm{C}, R_{\mathrm{f}}=0.86, \mathrm{CHCl}_{3}: \mathrm{MeOH}\right.$, 95:5). ${ }^{1} \mathrm{H}$ NMR (300 MHz, acetone- $\left.d_{6}\right) \delta(\mathrm{ppm}): 0.87(t, 6 \mathrm{H}$, $J=6.9 \mathrm{~Hz}, \mathrm{C}-7$ - and C-4'-OOC $\left.\left(\mathrm{CH}_{2}\right)_{14} \mathrm{CH}_{3}\right) ; 1.28(\mathrm{~s}, 44 \mathrm{H}$, C-7- and C-4'-OOC $\left.\left(\mathrm{CH}_{2}\right)_{3}\left(\mathrm{CH}_{2}\right)_{11} \mathrm{CH}_{3}\right) ; 1.40(\mathrm{~m}, 4 \mathrm{H}, J=$ $6.9 \mathrm{~Hz}, \mathrm{C}-7$ - and C-4'-OOC $\left.\left(\mathrm{CH}_{2}\right)_{2} \mathrm{CH}_{2}\left(\mathrm{CH}_{2}\right)_{11} \mathrm{CH}_{3}\right) ; 1.59$ (d, $6 \mathrm{H}, J=1.2 \mathrm{~Hz}, \mathrm{CH}_{3}-4^{\prime \prime}$ and $\mathrm{CH}_{3}-5^{\prime \prime}$ ); 1.73 (kwintet, $4 \mathrm{H}$, $J=7.3 \mathrm{~Hz}, \mathrm{C}-7-$ and C-4'-OOCCH$\left.{ }_{2} \mathrm{CH}_{2}\left(\mathrm{CH}_{2}\right)_{12} \mathrm{CH}_{3}\right) ; 2.60$ and 2.64 (two $t, 4 \mathrm{H}, J=7.3 \mathrm{~Hz}, \mathrm{C}-7$ - and $\mathrm{C}-4^{\prime}-\mathrm{OO}-$ $\left.\mathrm{CCH}_{2}\left(\mathrm{CH}_{2}\right)_{13} \mathrm{CH}_{3}\right) ; 2.78(\mathrm{dd}, 1 \mathrm{H}, J=16.3 \mathrm{~Hz}, J=3.0 \mathrm{~Hz}$, CH-3); 3.07 (dd, $1 \mathrm{H}, J=16.3 \mathrm{~Hz}, J=12.9 \mathrm{~Hz}, \mathrm{CH}-3$ ); 3.19 $\left(\mathrm{d}, 2 \mathrm{H}, J=6.7 \mathrm{~Hz}, \mathrm{CH}_{2}-1^{\prime \prime}\right) ; 3.80$ (s, 3H, C-5- $-\mathrm{OCH}_{3}$ ); 5.08 $\left(t_{\text {sept }}, 1 \mathrm{H}, J=6.7 \mathrm{~Hz}, J=1.2 \mathrm{~Hz}, \mathrm{CH}-2^{\prime \prime}\right) ; 5.60(\mathrm{dd}, 1 \mathrm{H}$, $J=12.9 \mathrm{~Hz}, J=3.0 \mathrm{~Hz}, \mathrm{CH}-2) ; 6.47$ (s, $1 \mathrm{H}, \mathrm{CH}-6) ; 7.20(\mathrm{~d}$, $2 \mathrm{H}, J=8.5 \mathrm{~Hz}, \mathrm{CH}-3^{\prime}$ and $\left.\mathrm{CH}-5^{\prime}\right) ; 7.62(\mathrm{~d}, 2 \mathrm{H}, J=8.5 \mathrm{~Hz}$, CH-2 $2^{\prime}$ and CH-6'). IR (KBr) cm ${ }^{-1}: 3184,2919,2850,1759$, 1688, 1589, 1510, 1468, 1376, 1265, 1139, 1102, 844, 721. $\mathrm{C}_{53} \mathrm{H}_{82} \mathrm{O}_{7}$ (831.24): calcd. C 76.58, H 9.94; found C 76.48, H 10.14 .

\section{Demethylation of isoxanthohumol derivatives}

General procedure Each time $50 \mathrm{mg}$ of compounds (4-10) were demethylated.

A solution of $\mathrm{I}_{2}$ (3 eq., $99.5 \mathrm{mg}, 0.393 \mathrm{mmol}$ ) in anhydrous $\mathrm{Et}_{2} \mathrm{O}(3.5 \mathrm{ml})$ and $\mathrm{Mg}$ (6 eq., $19.1 \mathrm{mg}, 0.786 \mathrm{mmol}$ ), taken in the round-bottomed flask and protected from light, was stirred at room temperature until the reaction mixture turned colorless $(1.5 \mathrm{~h})$. The resulting mixture of magnesium iodide etherate was separated from unreacted $\mathrm{Mg}$ and transferred via syringe under $\mathrm{N}_{2}$ into the two-neck flask (50 ml), equipped with condenser, containing $50 \mathrm{mg}$ of substrate [4 (1 eq., $0.131 \mathrm{mmol})-10]$ in anhydrous THF 
$(9 \mathrm{ml})$. The reaction mixture was stirred and refluxed for $12 \mathrm{~h}$ and afterward the solvent was evaporated under reduced pressure. Then, $1 \mathrm{ml}$ of THF and saturated solution of $\mathrm{NH}_{4} \mathrm{Cl}(10 \mathrm{ml})$ were added and the whole mixture was extracted with $\mathrm{CH}_{2} \mathrm{Cl}_{2}(3 \times 5 \mathrm{ml})$. The combined extracts were dried over anhydrous $\mathrm{Na}_{2} \mathrm{SO}_{4}$ and the solvent was removed under reduced pressure to give crude product. After purification by column chromatography on silica gel (see Table 1) the products (11-15) were obtained.

7,4'-di-O-methyl-8-prenylnaringenin (11) Yield 61.3\%, $\mathrm{mp}=105-107^{\circ} \mathrm{C}, R_{\mathrm{f}}=0.32\left(\mathrm{CHCl}_{3}\right.$ :hexane, $\left.7: 3\right)$, lightwhite solid. The ${ }^{1} \mathrm{H}$ NMR and IR spectroscopic data were in agreement with those reported in the literature (Cano et al., 2006; Siddiqui et al., 2003).

7-O-pentyl-8-prenylnaringenin (12) Yield 84.8\%, $\mathrm{mp}=$ $132-134^{\circ} \mathrm{C}, R_{\mathrm{f}}=0.67\left(\mathrm{CHCl}_{3}: \mathrm{MeOH}\right), 97: 3$, white crystals. ${ }^{1} \mathrm{H}$ NMR $\left(300 \mathrm{MHz}\right.$, acetone- $\left.d_{6}\right) \delta(\mathrm{ppm}): 0.93(t, 3 \mathrm{H}$, $\left.J=7.3 \mathrm{~Hz}, \quad \mathrm{C}-7-\mathrm{O}\left(\mathrm{CH}_{2}\right)_{4} \mathrm{CH}_{3}\right) ; 1.41 \quad$ (m, 2H, C-7$\left.\mathrm{O}\left(\mathrm{CH}_{2}\right)_{3} \mathrm{CH}_{2} \mathrm{CH}_{3}\right) ; 1.49\left(\mathrm{~m}, 2 \mathrm{H}, \mathrm{C}-7-\mathrm{O}\left(\mathrm{CH}_{2}\right)_{2} \mathrm{CH}_{2} \mathrm{CH}_{2} \mathrm{CH}_{3}\right)$; $1.61\left(\mathrm{~d}, 6 \mathrm{H}, J=1.4 \mathrm{~Hz}, \mathrm{CH}_{3}-4^{\prime \prime}\right.$ and $\left.\mathrm{CH}_{3}-5^{\prime \prime}\right) ; 1.82(\mathrm{~m}, 2 \mathrm{H}$, C7- $\left.\mathrm{OCH}_{2} \mathrm{CH}_{2}\left(\mathrm{CH}_{2}\right)_{2} \mathrm{CH}_{3}\right) ; 2.79(\mathrm{dd}, 1 \mathrm{H}, \quad J=17.0 \mathrm{~Hz}$, $J=3.0 \mathrm{~Hz}, \mathrm{CH}-3) ; 3.16(\mathrm{dd}, 1 \mathrm{H}, J=17.0 \mathrm{~Hz}, J=12.6 \mathrm{~Hz}$, CH-3); 3.22 (d, 2H, J=7.2 Hz, $\left.\mathrm{CH}_{2}-1^{\prime \prime}\right) ; 4.08(t, 2 \mathrm{H}$, $\left.J=6.3 \mathrm{~Hz}, \mathrm{C}-7-\mathrm{OCH}_{2}\left(\mathrm{CH}_{2}\right)_{3} \mathrm{CH}_{3}\right) ; 5.15\left(t_{\text {sept }}, 1 \mathrm{H}, J=\right.$ $\left.7.2 \mathrm{~Hz}, J=1.4 \mathrm{~Hz}, \mathrm{CH}-2^{\prime \prime}\right) ; 5.46(\mathrm{dd}, 1 \mathrm{H}, J=12.6 \mathrm{~Hz}$, $J=3.0 \mathrm{~Hz}, \mathrm{CH}-2) ; 6.12$ (s, 1H, CH-6); 6.90 (d, 2H, $J=8.5 \mathrm{~Hz}, \mathrm{CH}-3^{\prime}$ and $\left.\mathrm{CH}-5^{\prime}\right) ; 7.41(\mathrm{~d}, 2 \mathrm{H}, J=8.5 \mathrm{~Hz}, \mathrm{CH}-$ $2^{\prime}$ and $\left.\mathrm{CH}-6^{\prime}\right) ; 8.51$ (s, 1H, C-4'-OH); 12.24 (s, 1H, C-5-OH). IR (KBr) cm ${ }^{-1}: 3260,2955,2926,2855,1638,1616,1592$, 1520, 1467, 1364, 1229, 1094, 832. $\mathrm{C}_{25} \mathrm{H}_{30} \mathrm{O}_{5}$ (410.51): calcd. C 73.15, H 7.37; found C 73.32, H 7.54.

7,4'-Di-O-allyl-8-prenylnaringenin (13) Yield 78.9\%, $\mathrm{mp}=103-105^{\circ} \mathrm{C}, R_{\mathrm{f}}=0.84 \quad\left(\mathrm{CHCl}_{3}: \mathrm{MeOH}, 99.3: 0.7\right)$, pale yellow solid. ${ }^{1} \mathrm{H} \mathrm{NMR}\left(300 \mathrm{MHz}\right.$, acetone- $\left.d_{6}\right) \delta(\mathrm{ppm})$ : $1.60\left(\mathrm{~d}, 6 \mathrm{H}, J=1.3 \mathrm{~Hz}, \mathrm{CH}_{3}-4^{\prime \prime}\right.$ and $\left.\mathrm{CH}_{3}-5^{\prime \prime}\right) ; 2.82(\mathrm{dd}, 1 \mathrm{H}$, $J=17.1 \mathrm{~Hz}, J=3.1 \mathrm{~Hz}, \mathrm{CH}-3) ; 3.18(\mathrm{dd}, 1 \mathrm{H}, J=17.1$ $\mathrm{Hz}, J=12.5 \mathrm{~Hz}, \mathrm{CH}-3)$; 3.24 (d, $\left.2 \mathrm{H}, J=7.2 \mathrm{~Hz}, \mathrm{CH}_{2}-1^{\prime \prime}\right)$; 4.59 and 4.65 (two ddd, $4 \mathrm{H}, J=5.1 \mathrm{~Hz}, J=1.7 \mathrm{~Hz}$, $J=1.5 \mathrm{~Hz}, \mathrm{C}-7$ - and C-4'-OCH$\left.{ }_{2} \mathrm{CH}=\mathrm{CH}_{2}\right) ; 5.16\left(t_{\text {sept }}, 1 \mathrm{H}\right.$, $\left.J=7.2 \mathrm{~Hz}, J=1.3 \mathrm{~Hz}, \mathrm{CH}-2^{\prime \prime}\right) ; 5.23-5.31$ (m, 2H, trans$\mathrm{C}-7$ - and trans-C-4'-OCH$\left.{ }_{2} \mathrm{CH}=\mathrm{CH}_{2}\right) ; 5.51(\mathrm{dd}, 1 \mathrm{H}$, $J=12.5 \mathrm{~Hz}, J=3.1 \mathrm{~Hz}, \mathrm{CH}-2)$; 5.39-5.48 (m, 2H, cis-C7- and cis-C-4'- $\left.\mathrm{OCH}_{2} \mathrm{CH}=\mathrm{CH}_{2}\right) ; 6.02-6.16$ (m, 2H, C-7- and $\left.\mathrm{C}-4^{\prime}-\mathrm{OCH}_{2} \mathrm{CH}=\mathrm{CH}_{2}\right) ; 6.12(\mathrm{~s}, 1 \mathrm{H}, \mathrm{CH}-6) ; 7.02(\mathrm{~d}, 2 \mathrm{H}$, $J=8.8 \mathrm{~Hz}, \mathrm{CH}-3^{\prime}$ and $\left.\mathrm{CH}^{-} 5^{\prime}\right) ; 7.50(\mathrm{~d}, 2 \mathrm{H}, J=8.8 \mathrm{~Hz}$, CH-2' and $\left.\mathrm{CH}-6^{\prime}\right)$. IR (KBr) $\mathrm{cm}^{-1}: 2967,2911,2857,1636$, 1587, 1517, 1448, 1378, 1255, 1178, 1118, 1021, 921, 829. $\mathrm{C}_{26} \mathrm{H}_{28} \mathrm{O}_{5}$ (420.51): calcd. C 74.26, H 6.71; found C 74.09, $\mathrm{H}$ 6.88 .

7,4'-Di-O-acetyl-8-prenylnaringenin (14) Yield $88.4 \%$, $\mathrm{mp}=139-140^{\circ} \mathrm{C}, R_{\mathrm{f}}=0.84\left(\mathrm{CHCl}_{3}: \mathrm{MeOH}, 98: 2\right)$, white solid. ${ }^{1} \mathrm{HNMR}$ and IR spectroscopic data were in agreement with those reported in the literature (Gester et al., 2001; Huempel et al., 2005; Metz and Schwab, 2007; Schaefer et al., 2005).

7,4'-Di-O-palmitoyl-8-prenylnaringenin (15) Yield $74.6 \%, \mathrm{mp}=67-69^{\circ} \mathrm{C}, R_{\mathrm{f}}=0.91$ (hexane: $\mathrm{Et}_{2} \mathrm{O}: \mathrm{MeOH}$, 5:5:0.1), white crystals. ${ }^{1} \mathrm{H}$ NMR $\left(300 \mathrm{MHz}\right.$, acetone- $\left.d_{6}\right) \delta$ (ppm): $0.87\left(t, 6 \mathrm{H}, J=6.9 \mathrm{~Hz}, \mathrm{C}-7-\right.$ and C-4'-OOC $\left(\mathrm{CH}_{2}\right)_{14}-$ $\left.\mathrm{CH}_{3}\right) ; 1.29\left(\mathrm{~s}, 44 \mathrm{H}, \mathrm{C}-7-\right.$ and $\mathrm{C}-4^{\prime}-\mathrm{OOC}\left(\mathrm{CH}_{2}\right)_{3}\left(\mathrm{CH}_{2}\right)_{11}-$ $\left.\mathrm{CH}_{3}\right) ; 1.40\left(\mathrm{~m}, 4 \mathrm{H}, J=6.9 \mathrm{~Hz}, \mathrm{C}-7-\right.$ and $\mathrm{C}-4^{\prime}-\mathrm{OOC}$ $\left.\left(\mathrm{CH}_{2}\right)_{2} \mathrm{CH}_{2}\left(\mathrm{CH}_{2}\right)_{11}-\mathrm{CH}_{3}\right) ; 1.60\left(\mathrm{~d}, 6 \mathrm{H}, J=1.3 \mathrm{~Hz}, \mathrm{CH}_{3}-4^{\prime \prime}\right.$ and $\mathrm{CH}_{3}-5^{\prime \prime}$ ); 1.73 (quintet, $4 \mathrm{H}, J=6.9 \mathrm{~Hz}, \mathrm{C}-7$ - and C-4'$\mathrm{OOCCH}_{2} \mathrm{CH}_{2}\left(\mathrm{CH}_{2}\right)_{12}-\mathrm{CH}_{3}$ ); 2.60 and 2.64 (two $t, 4 \mathrm{H}$, $J=7.4 \mathrm{~Hz}, \mathrm{C}-7$ - and C-4'-OOCCH$\left.{ }_{2}\left(\mathrm{CH}_{2}\right)_{13}-\mathrm{CH}_{3}\right) ; 2.96$ (dd, $1 \mathrm{H}, J=17.2 \mathrm{~Hz}, J=3.0 \mathrm{~Hz}, \mathrm{CH}-3) ; 3.17$ (d, 2H, $\left.J=6.8 \mathrm{~Hz}, \mathrm{CH}_{2}-1^{\prime \prime}\right) ; 3.32(\mathrm{dd}, 1 \mathrm{H}, J=17.2 \mathrm{~Hz}, J=$ $13.1 \mathrm{~Hz}, \mathrm{CH}-3) ; 5.07\left(t_{\text {sept }}, 1 \mathrm{H}, J=6.8 \mathrm{~Hz}, J=1.3 \mathrm{~Hz}, \mathrm{CH}-\right.$ $\left.2^{\prime \prime}\right) ; 5.71(\mathrm{dd}, 1 \mathrm{H}, J=13.1 \mathrm{~Hz}, J=3.0 \mathrm{~Hz}, \mathrm{CH}-2) ; 6.30(\mathrm{~s}$, $1 \mathrm{H}, \mathrm{CH}-6) ; 7.22$ (d, 2H, $J=8.5 \mathrm{~Hz}, \mathrm{CH}-3^{\prime}$ and $\left.\mathrm{CH}-5^{\prime}\right) ; 7.65$ $\left(\mathrm{d}, 2 \mathrm{H}, J=8.5 \mathrm{~Hz}, \mathrm{CH}-2^{\prime}\right.$ and $\left.\mathrm{CH}-6^{\prime}\right) ; 11.87$ (s, 1H, C-5$\mathrm{OH})$. IR (KBr) cm $\mathrm{cm}^{-1}$ : 3437, 2918, 2850, 1751, 1648, 1624, 1592, 1512, 1469, 1379, 1264, 1149, 1077, 840, 722. $\mathrm{C}_{52} \mathrm{H}_{80} \mathrm{O}_{7}$ (817.21): calcd. C 76.43, H 9.87; found C 76.22, H 10.01 .

\section{Antiproliferative activity}

The human cell lines of breast cancer (MCF-7), colon adenocarcinoma (HT-29), and leukemia (CCRF/CEM) were obtained from American Type Culture Collection (Rockville, Maryland, USA) and maintained in the Cell Culture Collection at the Institute of Immunology and Experimental Therapy, Wroclaw, Poland. The cells at the density of $10^{5} / \mathrm{ml}$ were cultivated in 96-well plates (Sarstedt, Germany) in $100 \mu \mathrm{l}$ of culture medium at $37^{\circ} \mathrm{C}$ in humid atmosphere containing $5 \%$ $\mathrm{CO}_{2}$. In the case of MCF-7 cell lines, the culture medium

Table 1 Eluents for column chromatography for the purification of compounds (11-15)

\begin{tabular}{llllll}
\hline Compound & $\mathbf{1 1}$ & $\mathbf{1 2}$ & $\mathbf{1 3}$ & $\mathbf{1 4}$ & $\mathbf{1 5}$ \\
\hline Eluent & $\mathrm{CHCl}_{3}:$ hexane $70: 30$ & $\mathrm{CHCl}_{3}: \mathrm{MeOH} 99.2: 0.8$ & $\mathrm{CHCl}_{3} 100$ & $\mathrm{CHCl}_{3}: \mathrm{MeOH}_{99.5: 0.5}$ & $\mathrm{CHCl}_{3}: \mathrm{Et}_{2} \mathrm{O} 90: 10$ \\
\hline
\end{tabular}


consisted of Eagle's medium (IIET, Wroclaw, Poland) with addition of $10 \%$ fetal bovine serum (FBS, Sigma-Aldrich Chemie GmbH, Steinheim, Germany), $100 \mu \mathrm{g} / \mathrm{ml}$ streptomycin (Jelfa, Jelenia Góra, Poland), $100 \mathrm{U} / \mathrm{ml}$ penicillin (Jelfa, Jelenia Góra, Poland), 2 mM L-glutamine (Gibco, Warsaw, Poland), $1.0 \mathrm{mM}$ sodium pyruvate, $1 \%$ amino acid, and $0.8 \mathrm{mg} / \mathrm{l}$ insulin. The cells of HT-29 line were cultured in the RPMI 1640 and Opti-MEM (1:1) (both from Gibco) medium with addition of $5 \% \mathrm{FBS}, 100 \mu \mathrm{g} / \mathrm{ml}$ streptomycin, $100 \mathrm{U} / \mathrm{ml}$ penicillin, $1 \mathrm{mM}$ sodium pyruvate, and $2 \mathrm{mM}$ L-glutamine. CCRF/CEM culture medium consisted RPMI 1640, 10\% FBS, $100 \mu \mathrm{g} / \mathrm{ml}$ streptomycin, $100 \mathrm{U} / \mathrm{ml}$ penicillin and $2 \mathrm{mM}$ L-glutamine.

The compounds were dissolved in acetone (1-4, 8, and 10) or absolute ethanol $(\mathbf{5 - 7}, \mathbf{9}, \mathbf{1 1 - 1 3})$ to the concentration of $10 \mathrm{mg} / \mathrm{ml}$, stored at $4{ }^{\circ} \mathrm{C}$, and diluted in the culture medium to obtain concentrations from 0.1 to $100 \mu \mathrm{g} / \mathrm{ml}$. The controls contained acetone or ethanol at the appropriate concentrations. The solutions of the synthesized compounds in $100 \mu \mathrm{l}$ of culture medium were added after $24 \mathrm{~h}$ of incubation. The sulphorhodamine B (SRB, Sigma-Aldrich Chemie GmbH, Steinheim, Germany) assay for MCF-7 and HT-29 cells and the 3-(4,5-dimethylthiazol-2-yl)-2, 5-diphenyltetrazolium (MTT) assay (Sigma-Aldrich, Germany) for CCRF/CEM cells were executed. Assays were performed after $72 \mathrm{~h}$ of continuous exposure of the cultivated cells to varying concentrations of test compounds according to the methods described by Skehan et al. (1990) and Marcinkowska et al. (1998), using a Multiskan RC photometer (Labsystems, Helsinki, Finland). The readings were recorded at 540 and $570 \mathrm{~nm}$, respectively. Each compound at all the concentrations was investigated in triplicates. Each set of experiments was repeated 3-5 times.

SRB assay

The cells were attached to the bottom of plastic wells by gently layering cold $50 \%$ trichloroacetic acid (TCA) on the top of the culture medium in each well. The plates were stored at $4^{\circ} \mathrm{C}$ for $1 \mathrm{~h}$ and washed five times with tap water. The cells fixed with TCA were treated for 30 min with $0.4 \%$ solution of sulforhodamine $\mathrm{B}$ in $1 \%$ acetic acid. Then, the cells were washed four times with $1 \%$ acetic acid. The protein-bound dye was extracted with $10 \mathrm{mM}$ unbuffered Tris base. Optical density $(\lambda=540 \mathrm{~nm})$ was determined in a microplate reader Multiskan RC photometer.

\section{MTT assay}

Culture medium was gently removed from each well and cells were incubated for $4 \mathrm{~h}$ at $37^{\circ} \mathrm{C}$ with $20 \mu \mathrm{l}$ MTT solution $(5 \mathrm{mg} / \mathrm{ml})$. Then, $80 \mu \mathrm{l}$ of the mixture that contained $67.5 \mathrm{~g}$ sodium dodecyl sulfate and $225 \mathrm{ml}$ dimethylformamide in $275 \mathrm{ml}$ distilled water were added. After $24 \mathrm{~h}$ crystals of formazan were solubilized and the optical densities of the samples were read on a Multiskan RC photometer at $570 \mathrm{~nm}$.

\section{Results and discussion}

\section{Chemistry}

The main goal of this research was investigation of the demethylation reaction of substituted isoxanthohumols (4-10) to provide 8-prenylnaringenins (11-15). The investigated reactions are shown in Fig. 1 and the results are summarized in Table 2 .

Fig. 1 Synthesis of the isoxanthohumol derivatives (4-10) and 8-prenylnaringenin derivatives (11-15) from isoxanthohumol (2)

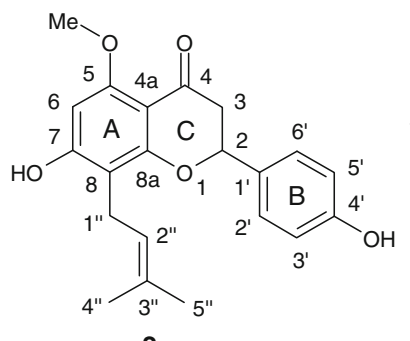

2

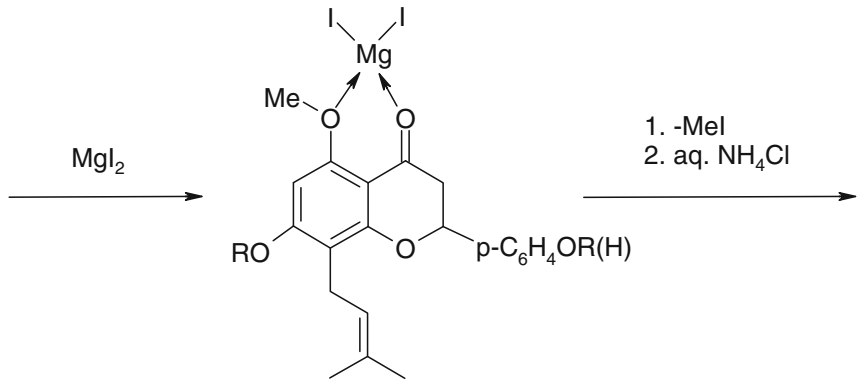

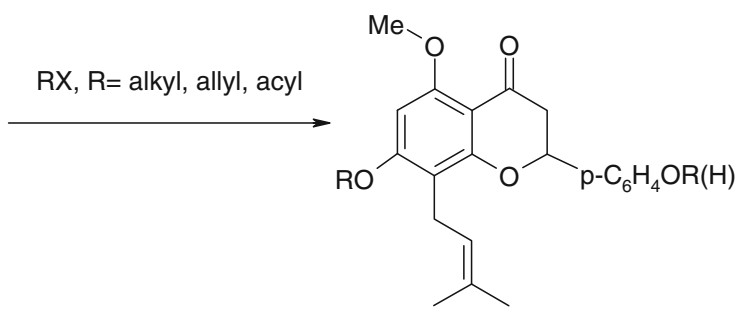

4 - 10

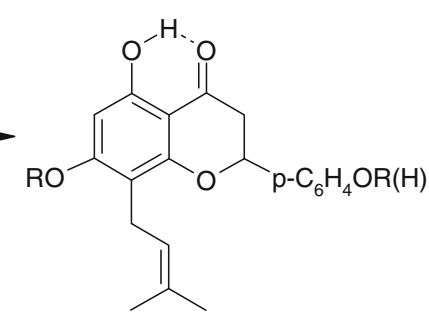

$11-15$ 
Table 2 Synthesis of 7-O- and 4'-O-substituted isoxanthohumols (4-10), their demethylation to 8-prenylnaringenins (11-15) and antiproliferative activity in vitro

\begin{tabular}{|c|c|c|c|c|c|c|c|c|}
\hline \multirow[t]{2}{*}{ Entry } & \multirow[t]{2}{*}{ Substrate } & \multirow[t]{2}{*}{ Product } & \multirow[t]{2}{*}{$\left.\operatorname{Yield}^{[\mathrm{a}]}[\%)\right]$} & \multirow[t]{2}{*}{$7-O-\mathrm{R}$} & \multirow[t]{2}{*}{$4^{\prime}-O-\mathrm{R}$} & \multicolumn{3}{|c|}{ Cell line/ID ${ }_{50}(\mu \mathrm{g} / \mathrm{ml}) \pm \mathrm{SD}$} \\
\hline & & & & & & MCF-7 & HT-29 & CCRF/CEM \\
\hline & - & 1 & - & - & - & $4.7 \pm 0.6$ & $3.8 \pm 0.6$ & $4.1 \pm 0.5$ \\
\hline & 1 & 2 & - & - & - & $9.4 \pm 0.4$ & $32.6 \pm 0.3$ & $18.2 \pm 1.9$ \\
\hline & 2 & 3 & - & - & - & $19.4 \pm 1.9$ & $33.2 \pm 0.8$ & $24.2 \pm 1.4$ \\
\hline $1 \mathbf{a}$ & 2 & 4 & 69.4 & $\mathrm{Me}$ & $\mathrm{Me}$ & $6.6 \pm 0.6$ & $6.0 \pm 1.2$ & $5.0 \pm 1.7$ \\
\hline $1 \mathbf{b}$ & 2 & 5 & 8.8 & $\mathrm{Me}$ & $\mathrm{H}$ & Not tested & Not tested & Not tested \\
\hline $2 \mathbf{a}$ & 2 & 6 & 27.6 & Pentyl & $\mathrm{H}$ & $8.3 \pm 1.2$ & $6.9 \pm 0.8$ & $5.4 \pm 0.9$ \\
\hline $2 \mathbf{b}$ & 2 & 7 & 13.6 & Pentyl & Pentyl & $7.1 \pm 0.6$ & $8.2 \pm 1.3$ & $4.3 \pm 0.7$ \\
\hline 3 & 2 & 8 & 81.2 & Allyl & Allyl & $5.2 \pm 0.1$ & $6.2 \pm 1.1$ & $2.7 \pm 0.5$ \\
\hline 4 & 2 & 9 & 74.1 & $\mathrm{Ac}$ & $\mathrm{Ac}$ & $16.9 \pm 2.3$ & $32.1 \pm 0.7$ & $23.3 \pm 1.1$ \\
\hline 5 & 2 & 10 & 81.6 & Palmitoyl & Palmitoyl & Negative & Negative & Negative \\
\hline 6 & 4 & 11 & 61.3 & $\mathrm{Me}$ & $\mathrm{Me}$ & $36.9 \pm 6.2$ & Negative & Negative \\
\hline 7 & 6 & 12 & 84.8 & Pentyl & $\mathrm{H}$ & $3.9 \pm 0.2$ & $10.0 \pm 2.9$ & $4.8 \pm 0.4$ \\
\hline 8 & 8 & 13 & 78.9 & Allyl & Allyl & Negative & Negative & Negative \\
\hline 9 & 9 & 14 & 88.4 & $\mathrm{Ac}$ & $\mathrm{Ac}$ & $28.0 \pm 2.6$ & $36.1 \pm 3.8$ & $37.0 \pm 3.5$ \\
\hline 10 & 10 & 15 & 74.6 & Palmitoyl & Palmitoyl & Negative & Negative & Negative \\
\hline
\end{tabular}

Negative Negative in the concentration used

${ }^{a}$ Isolated yield

Xanthohumol, the substrate in the isoxanthohumol synthesis, was isolated from carbon dioxide-extracted-hops (Marynka variety), purified and transformed into isoxanthohumol as described previously (Anioł et al., 2008).

As model substrates for demethylation, methyl, n-pentyl, allyl, acetyl, and palmitoyl derivatives of $\mathbf{2}$ were selected. They had different chain lengths. It was assumed that the reactivity of homologous series of compounds should be similar, as well as reactivity of monosubstituted isoxanthohumol derivatives in comparison to disubstituted. For this reason, alkylating and acylating agents were used in high quantity to obtain disubstituted derivatives of $\mathbf{2}$ as a goal of synthesis.

Methyl ethers (4 and 5) were synthesized using excess of methyl iodide with 69.4 and $8.8 \%$ yield, respectively (Table 2, Entries 1a and 1b). During the course of reaction, it was observed that the formation of 7-O-methyl compound (5), which was methylated to get a dimethyl compound (4). There was a characteristic shift of the signal for C-6 proton of substrate (2) from 6.21 to $6.36 \mathrm{ppm}$ for compound (5) on the NMR spectrum. It was caused by the substitution of $\mathrm{C}-7-\mathrm{OH}$ group by a methoxy group. The chemical shifts of C- $3^{\prime}-$, C-5'- and C-2'-, C- $6^{\prime}$-protons were exactly the same in both the compounds $(\delta=6.89$ and $7.38 \mathrm{ppm}$, respectively). The formation of products of cleavage of $\mathrm{C}$ ring leading to xanthohumol derivatives, as reported for methylation of 8-prenylnaringenin with $\mathrm{Me}_{2} \mathrm{SO}_{4}$ (Jain et al., 1978). In case of prenylation (Table 2, Entries $\mathbf{2 a}$ and $\mathbf{2 b}$ ), the order of alkylation was the same as that of compounds (4 and 5). The first product, 7- $O$-pentylisoxanthohumol (6) was formed with $27.6 \%$ yield $\left(\delta=6.34\right.$ (CH-6), $6.89\left(\mathrm{CH}-3^{\prime}, \mathrm{CH}_{-} 5^{\prime}\right)$ and $7.38 \mathrm{ppm}$ $\left(\mathrm{CH}-2^{\prime}, \mathrm{CH}_{-} 6^{\prime}\right)$, and 7, 4'-O-dipentylisoxanthohumol (7) with $13.6 \%$ yield. The best yield of alkylation was observed during the synthesis of the diallyl compound (8, Table 2, Entry 3). Diacyl compounds (9 and 10) were obtained with 74.1 and $81.6 \%$ yield, respectively (Table 2, Entries 4 and 5).

Demethylation reactions were carried out according to published procedure (Anioł et al., 2008). Each time $50 \mathrm{mg}$ of substrate was taken. The rest of the reagents were used proportionally in molar quantities. Demethylation of trimethoxy derivative (4) confirmed that the reaction of methyl-aryl ethers with magnesium iodide etherate occurred mainly at ortho-position in relation to acyl group. The main product of demethylation (11) was obtained with yield of $61.3 \%$ (Table 2, Entry 6) but during the reaction course, the formation of complicated mixture of by-products was observed, which was confirmed by TLC and HPLC. This reaction was not as clean as that of demethylation of isoxanthohumol (Anioł et al., 2008). The ${ }^{1} \mathrm{H}$ NMR spectrum of $\mathbf{1 1}$ showed the lack of signal of $\mathrm{C}-8-\mathrm{OCH}_{3}$ protons at $3.86 \mathrm{ppm}$, and the presence of signal at $12.25 \mathrm{ppm}$ for the proton of $\mathrm{C}-8-\mathrm{OH}$ group involved in a strong intramolecular hydrogen bond. A quite similar effect as above was observed for the rest of the synthesized 8-prenylnaringenin derivatives. All the spectra were recorded within $1-2 \mathrm{~h}$ after the sample preparation in 
acetone- $d_{6}$. When the spectrum was accumulated on the next day or later the signals for the hydroxyl protons disappeared because of the hydrogen deuterium exchange. Compound (11) was also isolated from Azadirachta indica (Siddiqui et al., 2003) and Esenbeckia berlandieri ssp. Acapulcensis (Cano et al., 2006). Substrate (4) used in the above reaction was present in hops in low quantity (Faltermeier et al., 2006; Oosterveld et al., 2002). For testing whether the demethylation depends on chain length of alkyl group, pentyl derivative of isoxanthohumol (6) was synthesized.

Demethylation of 7-O-pentylisoxanthohumol (6) to product (12) occurred with high yield of $84.8 \%$ (Table 2, Entry 7).

Cleavage of allyl ethers of alcohols and phenols was observed using lewis acids such as the $\mathrm{CeCl}_{3}-\mathrm{NaI}$ system (Bartoli et al., 2001; Thomas et al., 1999). Compound (8) was synthesized to check whether its demethylation was affected by deallylation. There was a possibility that $\mathrm{MgI}_{2}$, composed with magnesium (typical Lewis acid) and iodine (strong nucleophile) could be similar in action to $\mathrm{CeCl}_{3}-\mathrm{NaI}$ system. We did not observe the allyl-aryl ether cleavage and the desired product (13) were obtained with good $78.9 \%$ yield (Table 2, Entry 7). As in the case of alkyl ethers of isoxanthohumol, for testing whether the yield of demethylation depends on chain length of acyl group, diacetyl and dipalmitoyl derivatives of isoxanthohumol (9 and 10) were synthesized. These compounds, as representatives of esters, commonly applied as prodrugs, underwent demethylation with magnesium iodide etherate (Table 2, Entries 9 and 10). The products, 8-prenylnaringenins (14 and 15) were obtained with 88.4 and $74.6 \%$ yield, respectively. Thus, introduction of alkyl, allyl or acyl group into isoxanthohumol moiety did not significantly influence the demethylation reaction and all the synthesized compounds were stable during the course of reactions. Nevertheless, during the optimization of the isoxanthohumol demethylation (Anioł et al., 2008) to 8-prenylnaringenin the instability of reagents was observed, which could be associated with the known low stability of flavonoids.

Investigations conducted by a group of Wilhelm and Wessjohann (2006) showed that demethylation of such compounds as isoxanthohumol was very difficult to carry out. Among the 17 demethylating agents only $\mathrm{Sc}(\mathrm{OTf})_{3} / \mathrm{KI}$ system worked with high yield. Our previous investigations demonstrated that this system could be replaced with $\mathrm{MgI}_{2} \times 2 \mathrm{Et}_{2} \mathrm{O}$ to obtain 8-prenylnaringenin with $93 \%$ of yield. Now, we showed that this cheap, non toxic, easy to prepare and use agent could be applied in demethylation of acyl, alkyl, and allyl derivatives of isoxanthohumol.

Antiproliferative activity, in vitro

The synthesized compounds were examined for their antiproliferative activity in vitro against the human cell lines of breast cancer (MCF-7), colon adenocarcinoma (HT-29), and leukemia (CCRF/CEM). The results presented in Table 2 are expressed as the concentration in $\mu \mathrm{g} / \mathrm{ml}$ leading to $50 \%$ inhibition of tumor cells proliferation

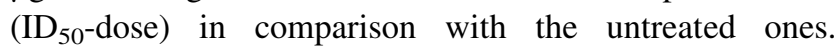
Acetone or ethanol, which was used as solvents, did not show any inhibitory effect on cell proliferation, even in the largest concentrations used. Xanthohumol (1), isoxanthohumol (2), and 8-prenylnaringenin (3), studied previously against selected tumor cell lines (Brunelli et al., 2007, 2009; Monteiro et al., 2007; Zanoli and Zavatti, 2008), were used as reference compounds. The two newly synthesized compounds ( 8 and 12) exhibited higher antiproliferative activity than the most active xanthohumol (1) against CCRF/CEM $(2.7 \mu \mathrm{g} / \mathrm{ml})$ and MCF-7 $(3.9 \mu \mathrm{g} / \mathrm{ml})$ cell lines and approaching the cytotoxic activity criterion $\mathrm{ID}_{50} \leq 4 \mu \mathrm{g} / \mathrm{ml}$ for new anticancer synthetic substances. The conducted investigations showed that, 7,4'-di- $O$ methyl-, 7,4'-di- $O$-pentyl-, and 7,4'-di- $O$-allyl- derivatives of isoxanthohumol $(\mathbf{4}, \mathbf{7}, \mathbf{8})$ were significantly more active than parental isoxanthohumol (2) $(9.4-32.6 \mu \mathrm{g} / \mathrm{ml})$ against all investigated cells $(2.7-6.6 \mu \mathrm{g} / \mathrm{ml})$. On the other hand, diacyl derivatives (9: $16.9-32.1 \mu \mathrm{g} / \mathrm{ml}$ and 10: $\mathrm{ID}_{50}>$ $100 \mu \mathrm{g} / \mathrm{ml}$ ) did not show any significant activity. Among the 8-prenylnaringenin derivatives, the most active compound was 7-O-pentyl-8-prenylnaringenin (12). This compound possessed the activity against the cells of MCF-7 $(3.9 \mu \mathrm{g} /$ $\mathrm{ml})$, HT-29 (10.0 $\mu \mathrm{g} / \mathrm{ml})$, and CCRF/CEM (4.8 $\mu \mathrm{g} / \mathrm{ml})$ more than three times higher than 8-prenylnaringenin (3), 19.4, $33.2,24.2 \mu \mathrm{g} / \mathrm{ml}$, respectively. The rest of the derivatives of 8-prenylnaringenin $(\mathbf{1 1}, \mathbf{1 3}-\mathbf{1 5})$ possessed low activity or were inactive $\left(\mathrm{ID}_{50}>100 \mu \mathrm{g} / \mathrm{ml}\right)$.

\section{Conclusion}

In conclusion, the presented simple methodology of demethylation of isoxanthohumol derivatives via the formation of magnesium iodide etherate, offers an easy transformation route for 8-prenylnaringenin derivatives synthesis using xanthohumol as a starting material, which can be applied to several functional groups. Although the yields obtained $(61.3-88.4 \%)$ were not as good as in case of demethylation of unsubstituted isoxanthohumol, the method was still easy, cheap and could be carried out in mild conditions. The synthesized compounds showed antiproliferative activity against the human cell lines of breast cancer (MCF-7), colon adenocarcinoma (HT-29), and leukemia (CCRF/CEM). The most active compound possessed activity of $2.7 \mu \mathrm{g} / \mathrm{ml}$ against leukemia cell lines. The developed demethylation protocol could be used in the synthesis of various potentially bioactive 8-prenylnaringenin derivatives and can be of use in the combinatorial 
chemistry to prepare libraries of such compounds. It would also help in proper utilization of the spent hops, the waste product of hop industry.

Acknowledgments Financial support for this study was provided by the Ministry of Sciences and Higher Education in Poland (project N N312 279634, years 2008-2011).

Open Access This article is distributed under the terms of the Creative Commons Attribution Noncommercial License which permits any noncommercial use, distribution, and reproduction in any medium, provided the original author(s) and source are credited.

\section{References}

Anioł M, Szymańska K, Żołnierczyk A (2008) An efficient synthesis of the phytoestrogen 8-prenylnaringenin from isoxanthohumol with magnesium iodide etherate. Tetrahedron 64:9544-9547

Bartoli G, Cupone G, Dalpozzo R, De Nino A, Maiuolo L, Marcantoni E, Procopio A (2001) Cerium-mediated deprotection of substituted allyl ethers. Synlett 12:1897-1900

Borrelli F, Ernst E (2010) Alternative and complementary therapies for the menopause. Maturitas 66:333-343

Böttner M (2008) Effects of long-term treatment with 8-prenylnaringenin and oral estradiol on the GH-IGF-1 axis and lipid metabolism in rats. J Endocrinol 198:395-401

Brunelli E, Minassi A, Appendino G, Moro L (2007) 8-prenylnaringenin, inhibits estrogen receptor- $\alpha$ mediated cell growth and induces apoptosis in MCF-7 breast cancer cells. J Steroid Biochem Mol Biol 107:140-148

Brunelli E, Pinton G, Chianale F, Graziani A, Appendino G, Moro L (2009) 8-prenylnaringenin inhibits epidermal growth factorinduced MCF-7 breast cancer cell proliferation by targeting phosphatidylinositol-3-OH kinase activity. J Steroid Biochem Mol Biol 113:163-170

Cano A, Espinoza M, Ramos CH, Delgado G (2006) New prenylated flavanones from Esenbeckia berlandieri ssp. Acapulcensis. J Mexican Chem Soc 50:71-75

Chadwick LR, Paul GF, Farnsworth NR (2006) The pharmacognosy of Humulus lupulusL. (hops) with an emphasis on estrogenic properties. Phytomedicine 13:119-131

Colgate EC, Miranda CL, Stevens JF, Bray TM, Ho E (2007) Xanthohumol, a prenylflavonoid derived from hops induces apoptosis and inhibits NF-kappaB activation in prostate epithelial cells. Cancer Lett 246:201-209

Cos P, Maes L, Vlietinck A, Pieters L (2008) Plant-derived compounds for chemotherapy of human immunodeficiency virus (HIV) infection; an update (1998-2007). Planta Med 74:1323-1337

Delmulle L, Bellahcene A, Dhooge W, Comhaire F, Roelens F, Huvaere K, Heyerick A, Castronovo V, De Keukeleire D (2006) Anti-proliferative properties of prenylated flavonoids from hops (Humulus lupulus L.) in human prostate cancer cell lines. Phytomedicine 13:732-734

Drenzek JG, Seiler NL, Jaskula-Sztul R, Rausch MM, Rose SL (2011) Xanthohumol decreases Notch1 expression and cell growth by cell cycle arrest and induction of apoptosis in epithelial ovarian cancer cell lines. Gynecol Oncol 122:396-401

Faltermeier A, Massinger S, Schulmeyr J (2006) Process for preparing high-purity xanthohumol-containing powder and use thereof. Patentinhaber: NATECO@ GmbH \& Co. KG German Patent Application DE 102006018988.4
Gester S, Metz P, Zierau O, Vollmer G (2001) An efficient synthesis of the potent phytoestrogens 8-prenylnaringenin and 6-(1, 1-dimethylallyl)naringenin by europium(III)-catalyzed Claisen rearrangement. Tetrahedron 57:1015-1018

Huempel M, Schleuning WD, Schaefer O, Isaksson P, Bohlmann R (2005) Use of 8-Prenylnaringenin for Hormone Replacement Therapy. European Patent Application EP 1524269 A1

Hyun JK, So-Hyun K, Bok YK, Ik-Soo L (2008) Microbial metabolism of the prenylated chalcone xanthohumol. Arch Pharm Res 31:1241-1246

Jacob C, Jamier V, Ba LA (2011) Redox active secondary metabolites. Curr Opin Chem Biol 2011(15):149-155

Jain AC, Gupta RC, Sarpal PD (1978) Synthesis of racemic 8-Cprenyl- $6^{\prime \prime}, 6^{\prime \prime}$-dimethylpyrano $\left(2^{\prime \prime}, 3^{\prime \prime}: 7,6\right)$ naringenin. Tetrahedron 34:3563-3567

Marcinkowska E, Kutner A, Radzikowski C (1998) Cell differentiating and anti-proliferative activity of side-chain modified analogues of 1,25-dihydroxyvitamin D3. J Steroid Biochem Mol Biol 67:71-78

Metz P, Schwab P (2007) Preparation of (2S)- and (2R)-8-prenylnaringenin, used in e.g. pharmaceuticals, comprises reducing racemic mixture of 8-prenylnaringenin derivative with formic acid and base, separating non-transferred enantiomer and splitting acyl residue. German Patent Application DE 10 2006032500

Monteiro R, Faria A, Azevedo I, Calhau C (2007) Modulation of breast cancer cell survival by aromatase inhibiting hop (Humulus lupulus L.) flavonoids. J Steroid Biochem Mol Biol 105:124-130

Okano J, Fujise Y, Abe R, Imamoto R, Murawaki Y (2011) Chemoprevention against hepatocellular carcinoma. Clin J Gastroenterol 4:185-197

Oosterveld A, Voragen AGJ, Schols HA (2002) Characterization of hop pectins shows the presence of an arabinogalactan-protein. Carbohydr Polym 49:407-413

Overk C, Guo J, Chadwick L, Main M, Lantvit D, Minassi A, Appendino G, Pauli GF, van Breemen R, Farnsworth N, Boltona J (2008) In vivo estrogenic comparisons of Trifolium pratense (red clover), Humulus lupulus (Hops), and the Pure compounds isoxanthohumol and 8-prenylnaringenin. Chem-Biol Interact 176:30-39

Schaefer O, Bohlmann R, Schleuning WD, Schulze-Forster K, Huempel M (2005) Development of a radioimmunoassay for the quantitative determination of 8-prenylnaringenin in biological matrices. J Agric Food Chem 53:2881-2889

Siddiqui BS, Ali ST, Rasheed M, Kardar MN (2003) Chemical constituents of the flowers of Azadirachta indica. Helv Chim Acta 86:2787-2796

Skehan P, Storeng R, Scudiero D (1990) New colorimetric cytotoxicity assay for anticancer-drug screening. J Nat Cancer Inst 82:1107-1112

Stevens JF, Page JE (2004) Xanthohumol and related prenylflavonoids from hops and beer to your good health. Phytohemistry 65:1317-1330

Stevens JF, Taylor AW, Nickerson GB, Ivancic M, Henning J, Haunold A, Deinzer ML (2000) Prenyl flavonoid variation in Humulus lupulus: distribution and taxonomic significance of xanthogalenol and 4'-O-methylxanthohumol. Phytochemistry 53:759-775

Thomas RM, Reddy GS, Iyengar DS (1999) An efficient and selective deprotection of allyl ethers by a $\mathrm{CeCl}_{3} \cdot 7 \mathrm{H}_{2} \mathrm{O}-\mathrm{NaI}$ system. Tetrahedron Lett 40:7293-7294

Wesołowska O, Wiśniewski J, Środa K, Krawczenko A, BielawskaPohl A, Paprocka M, Duś D, Michalak K (2010a) 8-Prenylnaringenin is an inhibitor of multidrug resistance-associated transporters P-glycoprotein and MRP1. Eur J Pharmacol 644:32-40

Wesołowska O, Wiśniewski J, Środa K, Krawczenko A, Bielawska-Pohl A, Paprocka M, Duś D, Michalak K (2010b) 8-Prenylnaringenin is 
an inhibitor of multidrug resistance-associated transporters P-glycoprotein and MRP1. Eur J Pharmacol 644:32-40

Wilhelm H, Wessjohann LA (2006) An efficient synthesis of the phytoestrogen 8-prenylnaringenin from xanthohumol by a novel demethylation process. Tetrahedron 62:6961-6966

Yamaguchi S, Takai M, Hanazome I, Okada Y, Kawase Y (1987)

Synthesis and structural studies of remirol. Bull Chem Soc Jpn 60:3603-3605
Yamaguchi S, Nedachi M, Yokoyama H, Hirai Y (1999) Regioselective demethylation of 2,6-dimethoxybenzaldehydes with magnesium iodide etherate. Tetrahedron Lett 40:7363-7365

Zanoli P, Zavatti M (2008) Pharmacognostic and pharmacological profile of Humulus lupulus L. J. Ethnopharmacol 116:383-396 